\title{
Primary Antiphospholipid Syndrome Presenting as HELLP Syndrome
}

\author{
A Clinical Pathology Conference Held by the Division of Rheumatology \\ at Hospital for Special Surgery
}

\author{
Diane George, MD • Lisa Vasanth, MD • Doruk Erkan, MD • Anne Bass, MD • \\ Jane Salmon, MD • Michael D. Lockshin, MD
}

Published online: 26 April 2007

(C) Hospital for Special Surgery 2007

\section{Case presentation}

A 29-year-old woman (gravida 1, para 0) was transferred to New York Presbyterian Hospital (NYPH) for the evaluation of complete vision loss in the left eye, which occurred 4 days after an emergency medical abortion because of hemolysis, elevated liver enzymes, and low platelets (HELLP) syndrome.

This previously healthy patient was admitted to an outside hospital at 21 weeks gestation with 1 day history of right upper quadrant pain, uncontrolled hypertension, hematemesis, and severe headache as well as severe proteinuria, hematuria, and bilateral lower extremity swelling. Her significant physical examination findings were blood pressure $210 / 105 \mathrm{mmHg}$, heart rate 125 beats/ min, temperature $37.7^{\circ} \mathrm{C}$, respiratory rate $20 / \mathrm{min}$, scleral icterus, bibasilar inspiratory crackles, right upper quadrant abdominal tenderness without rebound or guarding, and one plus edema of lower extremities. Her initial laboratory tests are shown in Table 1.

Based on the initial assessment, she was diagnosed with preeclampsia/HELLP syndrome. She was started on magnesium sulfate for seizure prophylaxis, furosemide for pulmonary edema, and was given multiple platelet transfusions for thrombocytopenia. She also received a 4-day course of dexamethasone ( $40 \mathrm{mg}$ daily) for the preeclamp-

\section{George, MD (ه)}

Department of Internal Medicine,

St Lukes-Roosevelt Hospital Center,

1000 10th Avenue, New York, NY 10019, USA

e-mail: dg2301@columbia.edu

L. Vasanth, MD.D. Erkan, MD.A. Bass, MD.J. Salmon, MD.M. D. Lockshin, MD

Department of Rheumatology,

Hospital for Special Surgery,

535 East 70th Street, New York, NY 10021, USA
sia/HELLP syndrome. Despite treatment, she had worsening hypertension, right upper quadrant pain, and thrombocytopenia and underwent a termination of her pregnancy on her fourth hospital day. Although her blood pressure and thrombocytopenia improved, 2 days after the termination, she complained of blurred vision. Magnetic resonance (MR) imaging of the head and orbits, and MR venography were negative. She lost all vision in the left eye within 2 days and was transferred to NYPH for further management.

Her medical history was unremarkable except for a prolonged activated partial thromboplastin time (aPTT), false positive rapid plasma reagin, and a positive antinuclear antibody (ANA) 1:640, which were discovered on prenatal screening tests. She had been prescribed low-dose aspirin (LDA) since the end of the first trimester, and she took prenatal vitamins. She had no known drug allergies, denied previous miscarriages, ovarian cysts, fibroids, sexually transmitted diseases, or abnormal Papanicolaou smears. Her family history was negative for systemic lupus erythematosus (SLE), other autoimmune diseases, thrombophilia, or coagulopathy. She was born in Ecuador and moved to the United States 2 years before this admission. She denied any smoking, alcohol, or illicit drug use.

Her review of systems were negative for alopecia, oral ulcers, malar rash, photosensitivity, seizures, Raynaud's disease, arthralgia, myalgia, dysphagia, constipation, shortness of breath, cough, paroxysmal nocturnal dyspnea, orthopnea, chest pain, palpitations, fever, chills, fatigue, weight loss, dysuria, or hematuria.

On arrival to NYPH, except for a moderately elevated blood pressure, she was hemodynamically stable. She had an emergent ophthalmologic evaluation with fluorescein angiography that revealed retinal artery and vein occlusion and she was started on heparin. Factor V Leiden (factor V gene P506) mutation was absent and the homocysteine level was $7.3 \mu \mathrm{mol} / 1$ (normal 3-14). Further laboratory studies are shown in Table 2. Within 2 days of admission to NYPH, her lactate dehydrogenase $(401 \mathrm{U} / \mathrm{l})$, platelet $\left(310 \times 10^{9} / \mathrm{l}\right)$, 
Table 1 Initial laboratory tests

\begin{tabular}{lll}
\hline & Result & Normal Values \\
\hline White blood cell & 17.8 & \\
Hemoglobin & 8.9 & $4.0-10.0 \times 10^{9} / \mathrm{l}$ \\
Platelets & 17 & $12-16 \mathrm{~g} / \mathrm{dl}$ \\
Mean corpuscular volume & 90.3 & $150-350 \times 10^{9} / \mathrm{l}$ \\
Reticulocyte count & 4.18 & $80-100 \mathrm{fl}$ \\
aPTT & 40.7 & $0.5-1.5 \%$ \\
Prothrombin time & 11.1 & $25-35 \mathrm{~s}$ \\
Fibrinogen & 483 & $11-13 \mathrm{~s}$ \\
Creatinine & 1.3 & $150-350 \mathrm{mg} / \mathrm{dl}$ \\
Glucose & 93 & $0.7-1.3 \mathrm{mg} / \mathrm{dl}$ \\
Albumin & 2.0 & $70-100 \mathrm{mg} / \mathrm{dl} \mathrm{fasting}$ \\
Total bilirubin & 2.5 & $3.5-5.5 \mathrm{~g} / \mathrm{dl}$ \\
Direct bilirubin & 1.0 & $0.3-1.2 \mathrm{mg} / \mathrm{dl}$ \\
Aspartate aminotransferase & 1,498 & $0-0.3 \mathrm{mg} / \mathrm{dl}$ \\
Alanine aminotransferase & 395 & $0-35 \mathrm{U} / \mathrm{l}$ \\
Alkaline phosphatase & 99 & $0-35 \mathrm{U} / \mathrm{l}$ \\
Lactate dehydrogenase & 4,070 & $36-92 \mathrm{U} / \mathrm{l}$ \\
Uric acid & 7.8 & $60-100 \mathrm{U} / \mathrm{l}$ \\
Total cholesterol & 258 & $2.5-8.0 \mathrm{mg} / \mathrm{dl}$ \\
Low density lipoprotein & 119 & $150-199 \mathrm{mg} / \mathrm{dl}$ \\
Triglycerides & 198 & $<130 \mathrm{mg} / \mathrm{dl}$ \\
Urine protein & Large & $250 \mathrm{mg} / \mathrm{dl}$ \\
Urine blood & & Negative \\
Negative \\
\hline
\end{tabular}

aspartate aminotransferase (40 U/1), and alanine aminotransferase (57 U/1) levels were significantly improved.

\section{Pathological discussion: placenta}

The physiological conversion of the decidual vessels of the lining of the endometrium to uteroplacental vessels is a unique transformation that does not occur in any other vessels. Through the process of implantation and dilatation, the uterine spiral arterioles, which are small muscular arteries, dilate into uteroplacental vessels with the destruction of the smooth muscle in the arteriolar wall. The end result is dilated uteroplacental vessels that do not respond to normal stimuli for vasoconstriction and remain open as conduits during pregnancy to supply blood to the fetus (Fig. 1).
However, this patient's placenta had extensive changes of malperfusion, hypoxia, and ischemia with lack of physiologic conversion of the decidua to uteroplacental tissue, thus resulting in a marked decidual vasculopathy. The smooth muscle walls of the arterioles remained and the ensuing small luminal area decreased the blood flow to the placenta (Fig. 2). Other luminal changes that were seen as part of the decidual vasculopathy include fibrinoid necrosis of the vessel wall and decidual vascular thrombosis (Fig. 3). Early ischemic changes included agglutination of the villi, meaning that these villi were no longer participating in maternal-fetal exchange (Fig. 4).

As a normal pregnancy nears term, the remaining viable villi decrease in size so that there are more aggregates of syncytial nuclei called syncytial knots. At term, approximately $30 \%$ of the villi contain syncytial knots. However, in this placenta of approximately 21 weeks, there were more syncytial knots than expected, and the villi were smaller than expected for gestational age (Fig. 5). This accelerated maturity of the villi was a compensatory mechanism of the placenta to increase the surface area available for oxygen exchange in the face of hypoxia. Whereas some parts of the placenta attempted to compensate for the hypoxia, in other areas, chronic hypoxia caused inadequate growth and propagation of the villi, thus resulting in terminal villus deficiency. There was $25 \%$ infarction of the placenta, which is characteristic of preeclampsia and pregnancy-induced hypertension [1, 2]. However, when found to this degree in early pregnancy other underlying factors such as HELLP syndrome or antiphospholipid syndrome (APS) should be considered. Other conditions that could result in these placental pathological findings are inherited thrombophilias and SLE.

\section{Clinical discussion: obstetrical}

This patient's presentation had a broadly differential diagnosis. Right upper quadrant tenderness during the second trimester of pregnancy may be because of hepatitis, pancreatitis, cholecystitis, or appendicitis. Hematemesis

Table 2 Further laboratory tests

\begin{tabular}{|c|c|c|c|}
\hline & At the Time of Admission & 9 Days After Admission & 137 Days After Admission \\
\hline ANA & $1: 80$ centromere & Not tested & $3+$ \\
\hline Anti-ds-DNA & Negative & Not tested & Not tested \\
\hline Anti-Ro/La/Sm/RNP & Negative & Not tested & Not tested \\
\hline C3 (N: 85-193 mg/dl) & $90 \mathrm{mg} / \mathrm{dl}$ & Not tested & 89 mg/dl \\
\hline $\mathrm{C} 4(\mathrm{~N}: 12-360 \mathrm{mg} / \mathrm{dl})$ & $<10 \mathrm{mg} / \mathrm{dl}$ & Not tested & $15 \mathrm{mg} / \mathrm{dl}$ \\
\hline Lupus anticoagulant ${ }^{\mathrm{a}}$ & Negative & Not tested & Not tested \\
\hline $\mathrm{aCL} \operatorname{IgG}(\mathrm{IU})$ & 16 & $>80$ & $>80$ \\
\hline aCL IgM (IU) & 7 & 5 & 11 \\
\hline $\mathrm{aCL} \operatorname{IgA}(\mathrm{IU})$ & 40 & 51 & 63 \\
\hline $\mathrm{a} \beta_{2}$ GPI IgG (IU) & Not tested & 50 & $>100$ \\
\hline $\mathrm{a} \beta_{2}$ GPI IgM (IU) & Not tested & $<10$ & 11 \\
\hline $\mathrm{a} \beta_{2}$ GPI IgA (IU) & Not tested & 26 & 62 \\
\hline
\end{tabular}

ANA=antinuclear antibody, Anti-ds-DNA=antibodies to double-stranded DNA, C3=complement 3, C4=complement 4, aCL=anticardiolipin antibodies, a $32 \mathrm{GPI}=$ anti- $\beta 2$-glycoprotein-I antibodies, $\mathrm{Ig}=$ immunoglobulin

${ }^{\mathrm{a}}$ The Lupus anticoagulant test was performed according to International Consensus standards [7]. 


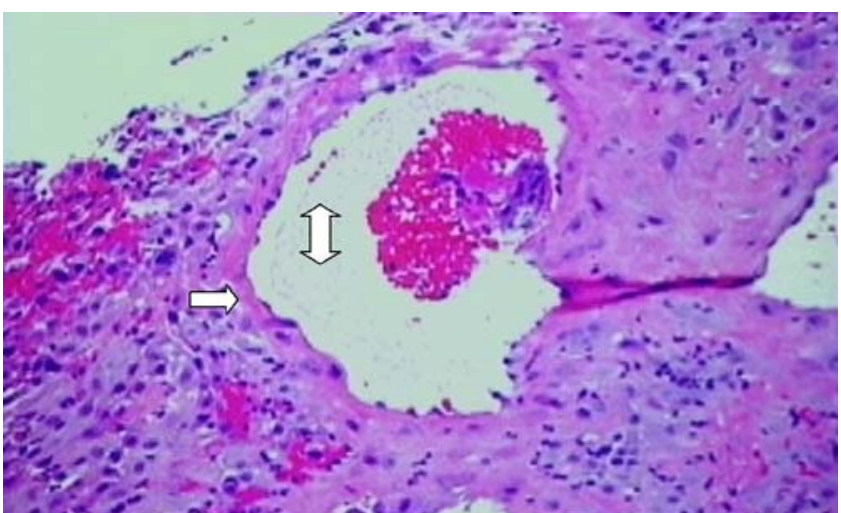

Fig. 1. Placental biopsy specimen demonstrating the normal physiological conversion of a decidual vessel. Note that the vessel has a large lumen (double-headed arrow) and that the vessel wall is composed of an endothelial layer but lacks a smooth muscle layer (singleheaded arrow)

may indicate severe gastritis, or if coupled with hematuria it could indicate either hemolytic uremic syndrome or thrombotic thrombocytopenic purpura. Because her ANA test was positive, SLE or another autoimmune disease was also a possibility. In this patient, the presence of preeclampsia with coexisting hemolysis, elevated liver function tests, and thrombocytopenia best corresponded with a diagnosis of HELLP syndrome.

The HELLP syndrome is part of a spectrum of hypertensive disorders of pregnancy, including preeclampsia, pregnancy-induced hypertension, and acute fatty liver of pregnancy. Preeclampsia is defined by hypertension, proteinuria with or without nondependent edema, whereas eclampsia is preeclampsia with seizures. HELLP syndrome is often associated with severe preeclampsia and eclampsia, and is considered a hallmark of critical illness [3]. Table 3 shows the diagnostic criteria for HELLP syndrome. In addition to the liver involvement, HELLP syndrome may have varying degrees of brain or kidney end-organ involvement.

The pathophysiology of preeclampsia is still unknown, but is postulated to be because of an improper trophoblastic

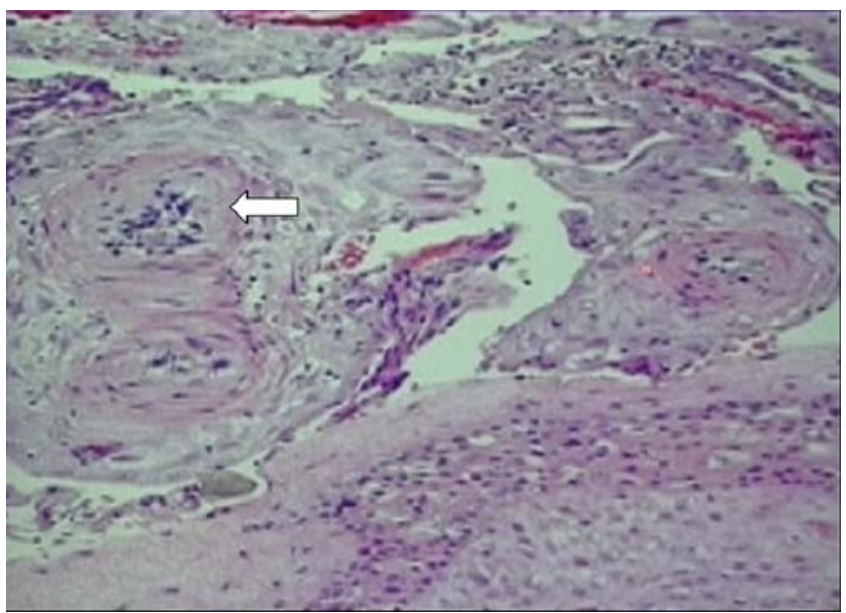

Fig. 2. Placental biopsy specimen demonstrating lack of physiological conversion resulting in decidual vasculopathy. Arrow indicates spiral arterioles with smooth muscular walls and small lumen

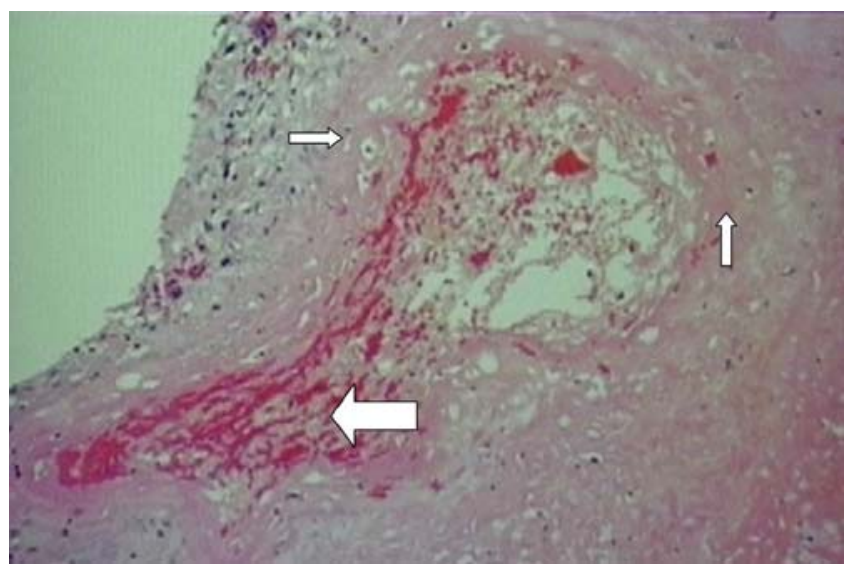

Fig. 3. Placental biopsy specimen demonstrating fibrinoid necrosis in the decidual vessel wall (smaller arrows) and thrombosis in decidual vessel (larger arrow)

invasion of the maternal uterine myometrium secondary to a triggering event. This abnormal placentation leads to placental ischemia causing the release of toxic factors, which enter the systemic circulation and result in endothelial injury [4]. This intimal injury stimulates fibrin deposition, which in turn, causes platelets to release vasoconstrictive substances such as thromboxane A2, and causes more platelet aggregation [5]. Eventually, there can be enough fibrin deposition to cause obstruction of the liver sinusoids. This ultimately leads to hepatocellular sequelae and end-organ damage.

The presentation of HELLP syndrome may be nonspecific, as with this patient, and can range from flu-like symptoms to convulsions, or gastrointestinal hemorrhage. Fifteen percent of HELLP syndrome occurs in the second trimester. In preeclampsia, patients tend to be of younger age and closer to term. It is important to diagnose HELLP syndrome as early as possible to prevent fetal complications such as placental abruption, perinatal asphyxia, extreme maturity, growth restriction, intraventricular hemorrhage, and respiratory distress syndrome. Maternal complications include pulmonary edema, liver hematoma, retinal detachment, acute renal failure, disseminated intravascular coagulation,

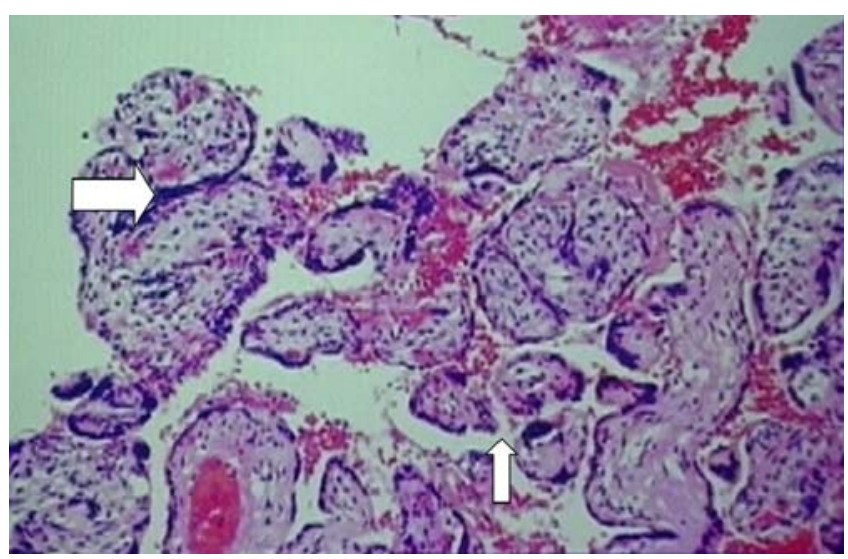

Fig. 4. Placental biopsy specimen demonstrating the early ischemic changes of villous agglutination (larger arrow). The smaller arrow indicates a normal intervillus space where maternal-fetal exchange takes place 


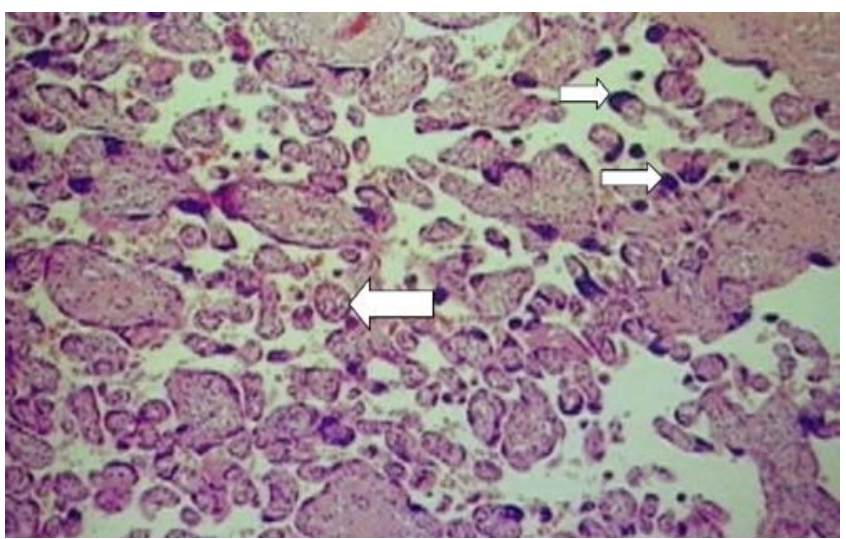

Fig. 5. Placental biopsy specimen demonstrating syncytial knots (smaller arrow), which are present in increased numbers for a 21week gestation. Larger arrow shows a small villus

cerebrovascular accident, acute respiratory distress syndrome, sepsis, and cerebral hemorrhage.

The treatment for severe preeclampsia and HELLP syndrome is immediate delivery, but unfortunately this patient's fetus was below the age of viability, and a medical termination was necessary. Delivery does not always result in cure; approximately $25 \%$ of HELLP syndrome patients are diagnosed postpartum.

\section{Clinical discussion: rheumatologic}

The combination of severe preeclampsia, HELLP syndrome, and an arterial and venous thrombosis narrows the differential diagnosis to a thrombophilia, vasculitis, or APS. Although many of the acquired and inherited thrombophilias can result in preeclampsia, uteroplacental insufficiency, and venous thrombosis, only hyperhomocysteinemia or APS would account for an arterial thrombosis. In this patient, the homocysteine level was found to be normal. She had persistently positive anticardiolipin antibody (aCL); thus, in the presence of retinal artery and vein thrombosis, she met the clinical and laboratory criteria for primary APS $[6,7]$. Marginally positive titers of ANA are not unusual in primary APS. A low C4 complement level, as seen in this patient, can be the result of severe preeclampsia.

This patient initially presented with both severe preeclampsia and HELLP syndrome. Severe preeclampsia is a recognized clinical manifestation of APS [8], and it is included under the pregnancy morbidity criteria of the updated Sapporo APS classification criteria (Table 4). The

Table 3 Diagnostic criteria for HELLP syndrome [27]

\begin{tabular}{ll}
\hline Criteria & Description \\
\hline Hemolysis & $\begin{array}{l}\text { Abnormal peripheral smear } \\
\text { Total bilirubin }>1.2 \mathrm{mg} / \mathrm{dl} \\
\text { Lactate dehydrogenase }>600 \mathrm{U} / 1 \\
\text { Espartate aminotransaminase/alanine } \\
\text { Eminotransferase }>70 \mathrm{U} / \mathrm{l}\end{array}$ \\
Low platelets & \begin{tabular}{l} 
Platelets $<100 \times 10^{9} / 1$ \\
\hline
\end{tabular} \\
\hline
\end{tabular}

Table 4 Revised Sapporo classification criteria for the APS [7]

Sapporo APS classification criteria

Clinical criteria

1. Vascular thrombosis

One or more clinical episodes of arterial, venous, or small vessel thrombosis in any tissue or organ

2. Pregnancy morbidity

(a) One or more unexplained deaths of a morphologically normal fetus at or beyond the 10th week of gestation, or

(b) One or more premature births of a morphologically normal neonate before the 34th week of gestation because of eclampsia, severe preeclampsia, or recognized features of placental insufficiency, or

(c) Three or more unexplained consecutive spontaneous abortions before the tenth week of gestation, with maternal anatomic or hormonal abnormalities and paternal and maternal chromosomal causes excluded.

Laboratory criteria

1. Lupus anticoagulant present in plasma on two or more occasions at least 12 weeks apart, detected according to the guidelines of the International Society on Thrombosis and Hemostasis

2. aCL of IgG and/or IgM isotype in serum or plasma present in medium or high titer (i.e., $>40$ GPL or MPL, or greater than the 99th percentile), on two or more occasions, at least 12 weeks apart, measured by a standardized ELISA.

3. Anti- $\beta_{2}$ glycoprotein-I antibody of $\operatorname{IgG}$ and/or IgM isotype in serum or plasma (in titer greater than the 99th percentile) present on two or more occasions, at least 12 weeks apart, measured by a standardized ELISA.

Definite APS is present if at least one of the clinical criteria and one of the laboratory criteria are met. Classification of APS should be avoided if less than 12 weeks or more than 5 years separate the positive aPL test and the clinical manifestation. In studies of populations of patients who have more than one type of pregnancy morbidity, investigators are strongly encouraged to stratify groups of subjects according to $\mathrm{a}, \mathrm{b}$, or $\mathrm{c}$ above.

ELISA=Enzyme-linked immunosorbent assay

connection between HELLP syndrome and APS is not clearly indicated in the literature. A retrospective study by Le Thi Thuong et al. [9] showed that HELLP syndrome may be the presenting manifestation of APS and that HELLP syndrome occurs at an earlier stage of gestation in APS than during a normal pregnancy. Another retrospective series of 75 pregnancies showed a $10.5 \%$ rate of APS in HELLP syndrome patients [10].

The rate of pregnancy loss in untreated APS is $80-90 \%$ $[11,12]$. Approximately $10-15 \%$ of healthy women with recurrent pregnancy losses have aPL [13], therefore establishing that the correct cause of pregnancy morbidity is important to optimize management. Other etiologies of early pregnancy loss should be first excluded, even in the presence aPL because embryonic pregnancy losses are common in the general population. Antiphospholipid syndrome is recognized to be a major contributor to late pregnancy losses [14]. As pregnancy and the postpartum period are prothrombotic states, management should focus on the prevention of pregnancy morbidity and maternal thrombotic complications.

Although management of aPL-positive patients is controversial because of limited well-designed controlled trials, several points need to be emphasized [14]: (1) corticosteroids are not indicated in the management of 
aPL-positive patients during pregnancy as they are associated with a high risk of complications such as gestational diabetes, amnionitis, and premature rupture of membranes; (2) aPL-related nongravid clinical manifestations represent a spectrum (asymptomatic, pregnancy morbidity only, vascular event, or catastrophic APS) and the management strategies during a pregnancy are different based on the position of aPL-positive patients on this spectrum; (3) LDA and prophylactic dose heparin are the standard therapy for patients fulfilling the updated Sapporo APS classification criteria based on pregnancy morbidity only; (4) LDA and therapeutic dose heparin are the standard therapy for patients fulfilling the updated Sapporo APS classification criteria based on a vascular event (this would be the case in the event of a subsequent pregnancy with this patient); and (5) LDA is usually given to asymptomatic medium-to-high titer aPL-positive patients during pregnancy, but there are no data to support this strategy (because pregnancy and the presence of aPL can be additive risk factors for vascular thrombosis, LDA therapy can be justified as it has minimal maternal or fetal side effects).

\section{Immunological discussion}

The exact mechanism by which aPL causes fetal loss is unknown, but it is believed to be mediated through abnormal placental function. In APS, decidual vasculopathy, as described in this patient, has been cited as an immediate cause of fetal loss by many investigations [15]; however, normal physiologic changes of the spiral arteries have also been reported in APS patients [16]. Antiphospholipid syndrome is associated with placental vascular thrombosis, decidual necrosis, and placental infarction [17, 18], as is seen in this patient's placental specimen, as well as inflammatory changes. Thus, altered coagulation and thrombosis in the uteroplacental circulation contributed to this pregnancy loss. Antiphospholipid antibodies are known to trigger the activation of endothelial cells and platelets $[19,20]$, but the pathogenic mechanisms leading to thrombosis and tissue injury are unclear.

Murine models demonstrate the direct pathogenic effect of aPL by the passive transfer of human immunoglobulin $\mathrm{G}$ (IgG) from high titer aPL positive patients' sera into pregnant mice, which result in fetal loss and growth retardation [21]. Further studies in this experimental model have shown that inhibition of complement activation by preventing the cleavage of complement 3 (C3), using Crry-Ig (an exogenously administered inhibitor of $\mathrm{C} 3$ activation), reduces fetal loss and growth retardation to levels seen in control mice [21]. Furthermore, Crry-Ig prevents C3 deposition within the deciduae and C3-deficient mice are resistant to fetal injury induced by aPL [22].

In a subsequent series of murine studies, APS induced by passive transfer of human aPL was prevented by treatment of mice with anti-C5 monoclonal antibodies and mice deficient in $\mathrm{C} 5$ were also protected. To distinguish the contribution of $\mathrm{C} 5 \mathrm{a}-\mathrm{C} 5 \mathrm{a}$ receptor (C5aR) interaction from those of the membrane attack complex
(C5b-9), mice deficient in C5aR and mice treated with C5aR antagonist peptide were also studied. Blockage of $\mathrm{C} 5 \mathrm{a}-\mathrm{C} 5 \mathrm{aR}$ interaction preserved pregnancies. These studies suggest that the C5-C5a receptor interactions are the critical intermediates linking pathogenic aPL to fetal damage $[23,24]$ and they implicate complement blockade, specifically at the level of $\mathrm{C} 5$ or $\mathrm{C} 5 \mathrm{aR}$ as a potential therapy for APS. Complement C5 monoclonal antibodies have been already utilized to treat other inflammatory conditions, and they could have a role in the prevention of fetal loss and pregnancy complications in APS.

Using pregnant APS mice, induced through passive transfer of human aPL, heparin has been shown to protect mice from aPL-induced pregnancy complications and prevent complement activation in vivo and in vitro. Interesting$\mathrm{ly}$, in the absence of a detectable anticoagulant effect, heparin still protects mice from aPL-induced pregnancy loss. Other anticoagulants, fondaparinux and hirudin, have not reduced the risk of aPL-induced pregnancy loss [25].

\section{Case presentation: long-term follow-up}

After 3 years of follow-up, the patient has remained anticoagulated with warfarin (international normalized ratio target 2.5-3.5), and she has not experienced any further thrombotic events. She has not regained vision in her left eye, and the optic disc remains pale. To date, she has had no further pregnancies. Although, the rate of thrombosis after the discontinuation of anticoagulation is high in APS patients [26], there is debate as to whether lifelong anticoagulation is necessary in APS patients, where a thrombotic risk factor, such as pregnancy in this case, can be identified as the trigger for clinical manifestations. The patient has consistently high positive titers of $\mathrm{aCL}$ and anti- $\beta_{2}$ glycoprotein-I antibodies but has not developed any clinical manifestations of SLE.

\section{Conclusion}

Antiphospholipid syndrome should be considered in the differential diagnosis of HELLP syndrome. Placental vascular thrombosis and inflammation, mediated by complement activation, increase the risk of growth restriction and fetal loss in APS. In addition to its anticoagulant role, heparin also reduces the risk of aPL-related pregnancy morbidities through the inhibition of complement activation. The specific modulation of the C5-C5a receptor interactions in APS may prove to be a new therapy in the future.

Acknowledgements The authors would like to thank Dr. Sriram C. Perni and Dr. Rebecca Baergen for their presentations and discussions during the clinical pathology conference.

\section{References}

1. Salafia CM, Pezzulo JC, Lopez-Zeno JA, Minior VK et al (1995) Placental pathologic features of preterm pre-eclampsia. Am J Obstet Gynecol 173(4): 1097-1105 
2. Shanklin DR, Sibai BM (1989) Ultrastructural aspects of preeclampsia I. Placental bed and uterine boundary vessels. Am J Obstet Gynecol 161(3): 735-741

3. Martin JN, Jr, Rinehart BK, May WL et al (1999) The spectrum of severe preeclampsia: Comparative analysis by HELLP (hemolysis, elevated liver enzyme levels, and low platelet count) syndrome classification. Am J Obstet Gynecol 180(6 Pt 1): 1373-1384

4. Curtin WM, Weinstein L (1999) A Review of HELLP syndrome: State of the Art. J Perinatol 19(2): 138-143

5. Baxter JK, Weinstein L (2004) HELLP syndrome: the state of the art. Obstet Gynecol Surv 59(12): 838-845

6. Wilson WA, Gharavi AE, Koike T et al (1999) International consensus statement on preliminary classification criteria for definite antiphospholipid syndrome: report of an international workshop. Arthritis Rheum 42(7): 1309-1311

7. Miyakis S, Lockshin MD, Atsumi $T$ et al (2006) International consensus statement on an update of the classification criteria for definite antiphospholipid syndrome (APS). J Thromb Haemost 4(2): 295-306

8. Branch DW, Silver RM, Blackwell JL, Reading JC, Scott JR (1992) Outcome of treated pregnancies in women with antiphospholipid syndrome: an update of the Utah experience. Obstet Gynecol 80(4): 614-620

9. Le Thi Thuong D, Tieulie N, Costedoat N et al (2005) The HELLP syndrome in the antiphospholipid syndrome: retrospective study of 16 cases in 15 women. Ann Rheum Dis 64(2): 273-278

10. Huong DL, Wechsler B, Bletry O et al (2001) A study of 75 pregnancies in patients with the antiphospholipid syndrome. J Rheumatol 28:2025-2030

11. Branch DW, Scott JR, Kochenour NK et al (1985) Obstetric complications associated with the lupus anticoagulant. N Engl J Med 313(21): 1322-1326

12. Rote NS, Walter A, Lyden TW et al (1992) Antiphospholipid antibodies-lobsters or red herrings. Am J Reprod Immunol 28(1): $31-37$

13. Sammaritano LR (2001) Update on the management of the pregnant patient with antiphospholipid antibody. Curr Rheumatol Rep 3(3): 213-221

14. Erkan D (2002) The relation between antiphospholipid syndrome-related pregnancy morbidity and non-gravid vascular thrombosis: review of the literature and management strategies. Curr Rheumatol Rep 4(5): 379-386
15. Magid MS, Kaplan C, Sammaritano LR et al (1998) Placental pathology in systemic lupus erythematosus: a prospective study. Am J Obstet Gynecol 179(1): 226-234

16. Van Horn JT, Craven C, Ward K et al (2004) Histologic features of placentas and abortion specimens from women with antiphospholipid and antiphospholipid like syndromes. Placenta 25(7): 642-648

17. Infante-Rievard C, David M, Gauthier R et al (1991) Lupus anticoagulants, anticardiolipin antibodies and fetal loss. N Engl J Med 325(15): 1063-1066

18. Rand JH, Wu XX, Andree HA et al (1997) Pregnancy loss in the antiphospholipid-antibody syndrome-a possible thrombogenic mechanism. N Engl J Med 337(3): 154-160

19. Simantov R, LaSala J, Lo SK et al (1995) Activation of cultured vascular endothelium by antiphospholipid antibodies. J Clin Invest 96:2211-2219

20. Pierangeli SS, Colden-Stanfield M, Liu X et al (1999) Antiphospholipid antibodies from antiphospholipid syndrome patients activate endothelial cells in vitro and in vivo. Circulation 99(15):1997-2002

21. Branch DW, Dudley DJ, Mitchell MD et al (1990) Immunoglobulin $\mathrm{G}$ fractions from patients with antiphospholipid antibodies cause fetal death in BABL/c mice: a model for autoimmune fetal loss. Am J Obstet Gynecol 163(1 Pt 1): 210-216

22. Holers VM, Girardi G, Guthridge GM et al (2002) Complement C3 activation is required for antiphospholipid antibody-induced fetal loss. J Exp Med 195(2): 211-220

23. Girardi G, Redecha P, Berman J et al (2004) Complement C5a receptors and neutrophils mediate fetal injury in the antiphospholipid syndrome. J Clin Invest 112(11): 1644-1654

24. Pierangeli SS, Girardi G, Vega-Ostertag M et al (2005) Requirement of activation of complement C3 and C5 for antiphospholipid antibody-mediated thrombophilia. Arthritis Rheum 52(7): 2120-2124

25. Girardi G, Redecha P, Salmon JE (2004) Heparin prevents antiphospholipid antibody-induced fetal loss by inhibiting complement activation. Nat Med 10(11): 1222-1226

26. Derekson RH, De Groot PG, Kater L, Nieuwenhuis HK (1993) Patients with Antiphospholipid antibodies and venous thrombosis should receive long term anticoagulant treatment. Ann Rheum Dis 52:689-692

27. Sibai BM (2004) Diagnosis, controversies, and management of the syndrome of hemolysis, elevated liver enzymes, and low platelet count. Obstet Gynecol 103(5 Pt 1): 981-991 